\title{
The Relationship Between Spiritual Health and Moral Sensitivity in Nursing Students of Hamadan University of Medical Sciences, 2017
}

\author{
Morteza Shamsizadeh ${ }^{1}$, Khodayar Oshvandi ${ }^{2}$, Kamran Alipour ${ }^{3}$, \\ Soraya Moamer ${ }^{4}$, Zahra Maghsoudi ${ }^{5^{*}}$
}

1. Instructor, Department of Medical Surgical Nursing, School of Nursing and Midwifery, Hamadan University of Medical Sciences, Hamadan, Iran

2. Professor, Department of Medical Surgical Nursing, Maternal and Child Care Research Center, Faculty of Nursing \& Midwifery, Hamadan University of Medical Sciences, Hamadan, Iran

3. Undergraduate Student of Nursing, Department of Medical Surgical Nursing, Student Research Committee, Hamadan University of Medical Sciences, Hamadan, Iran

4. PhD Student, Department of Biostatistics, Student Research Committee, Hamadan University of Medical Sciences, Hamadan, Iran

5. PhD Student in Nursing, Department of Medical Surgical Nursing, Student Research Committee, Hamadan University of Medical Sciences, Hamadan, Iran

\section{Article Info \\ Received: 2020/04/11; \\ Accepted: 2020/04/16; \\ Published Online: 2020/06/24 \\ $\underline{10.30699 / a j n m c .28 .2 .75}$}

Original Article

Use your device to scan and read the article online

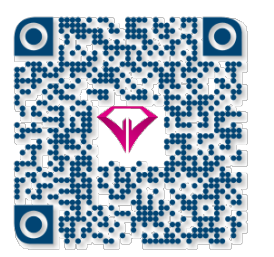

\section{ABSTRACT}

Introduction: Professional practice in nursing requires familiarity with the ethical issues of nursing profession and their sensitivity to patients. This sensitivity, as the basis of nursing ethics, should be considered from the beginning of nursing education. The degree of adherence to one's religion and the level of one's beliefs appear to be factors influencing moral sensitivity. The purpose of this study was to determine the relationship between spiritual health and moral sensitivity of nursing students.

Methods: In this descriptive-analytical study, 123 nursing students of Hamadan University of Medical Sciences were enrolled. Data were collected through questionnaire demographic information, Poultzan and Erison spiritual health questionnaire and moral sensitivity questionnaire. The data were analyzed by Chisquare and Pearson correlation coefficients using SPSS 21 software.

Results: The mean age of participants was $23.69 \pm 4.55$ years. Most nursing students had moderate spiritual health score and moral sensitivity. There was a positive and significant correlation between spiritual well-being with nursing students 'moral sensitivity $(\mathrm{r}=0.26, P=0.003)$, meaning that the higher the students' spiritual health score, the higher their moral sensitivity.

Conclusion: The results showed that spiritual health is an important parameter in promoting the moral sensitivity of nursing students. Therefore, promoting religious beliefs improves the health status of students and thus plays an important role in the implementation of effective patient care. In the promotion of their health, the spiritual aspect should also be taken into consideration.

Keywords: Nursing students, Spiritual health, Moral sensitivity

Copyright $($ ) 2020, This is an original open-access article distributed under the terms of the Creative Commons Attribution-noncommercial 4.0 International License which permits copy and redistribution of the material just in noncommercial usages with proper citation.

How to Cite This Article:

Shamsizadeh M, Oshvandi K, alipor K, Moamer S, Maghsoudi Z. The Relationship Between Spiritual Health and Moral Sensitivity in Nursing Students of Hamadan University of Medical Sciences, 2017. Avicenna J Nurs Midwifery Care. 2020; 28 (2) 
بررسى ارتباط سلامت معنوى با حساسيت اخلاقى دانشجويان ثرستارى

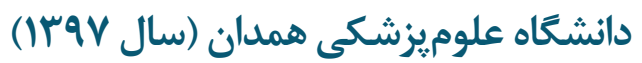

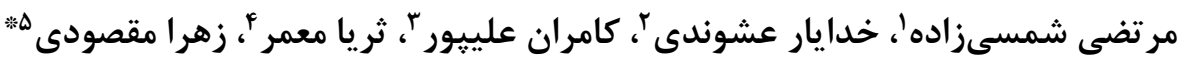

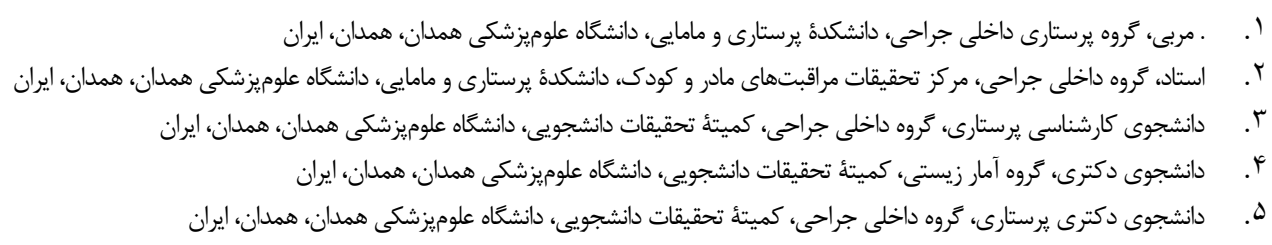

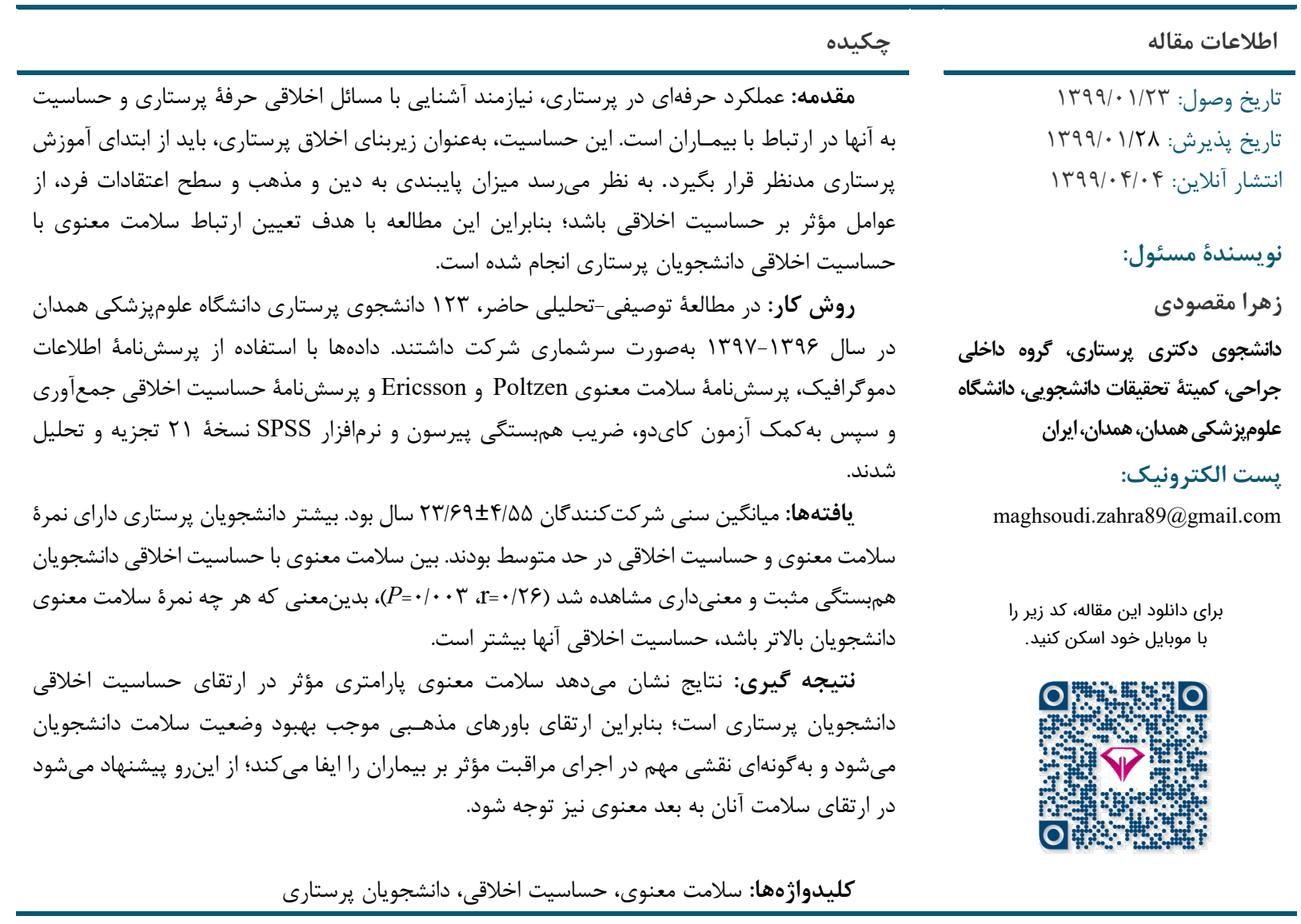

كافى نيز برخوردار باشند [F][]. در اين زمينه مىتوان كفت

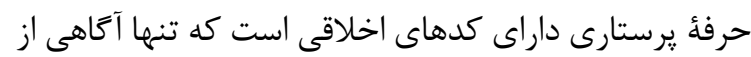

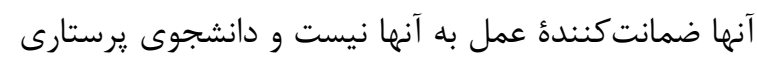

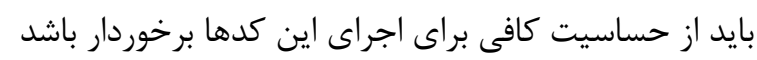

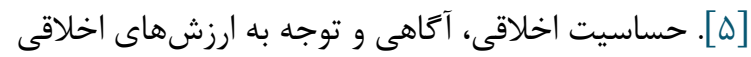
متناقض موجود در يك موقعيت و خودآكاهى فرد به نقش و و

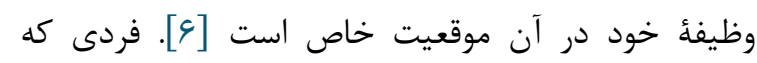

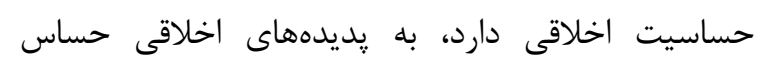

يرستاران بزرگترين گروه ارائه كنده خدمت در سيستم درمانى هستند و تأثير بسزايى بر كيفيت مراقبتهاى كراى

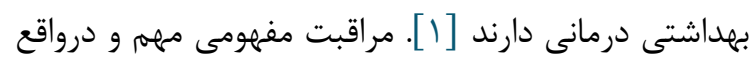

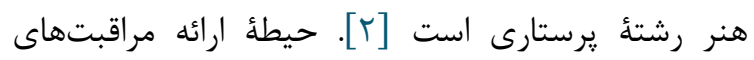

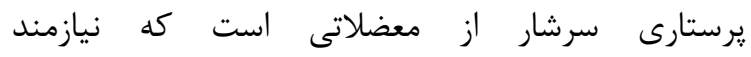

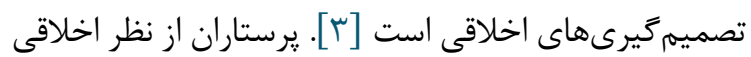

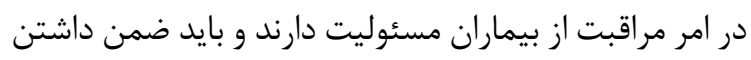

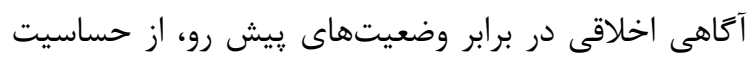


و سلامت وجودى مطرحكنندة ارتباط ما با ديخران، محيط و

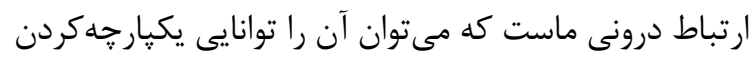

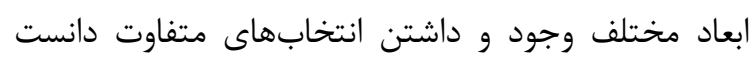
[19]. انسانهاى داراى سلامت معنوى مطلوب، رويكردى دان

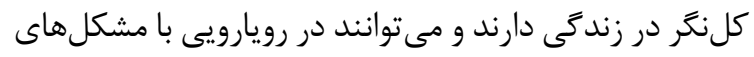

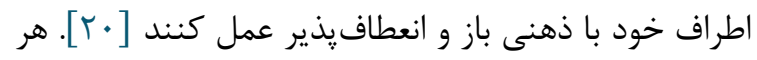

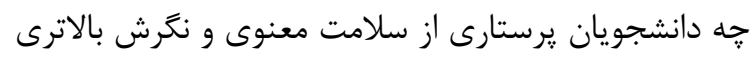

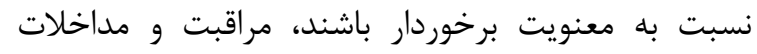
بهترى براى بيماران خود انجام مى دهند. Jalili و همكاران

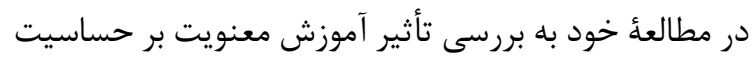

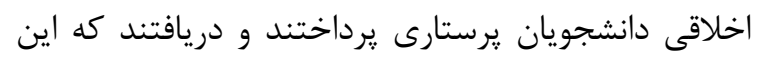

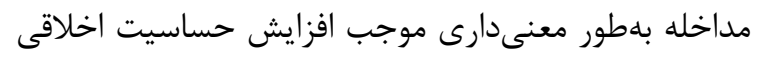

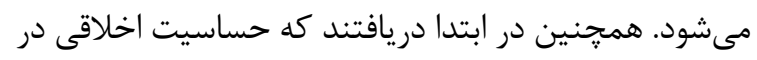

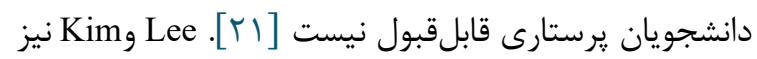
در مطالعئ خود دريافتند كه حساسيت اخلاقى دانشجويان

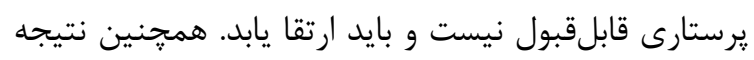

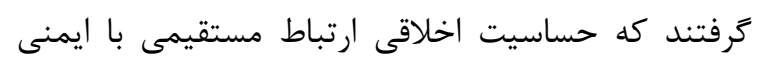

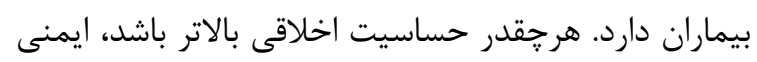

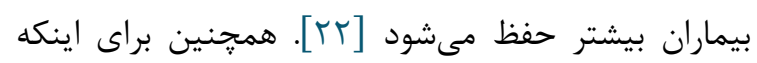

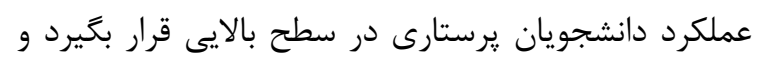

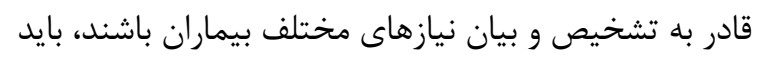

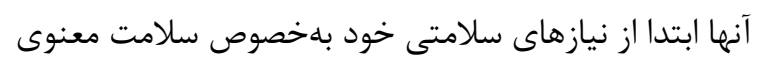

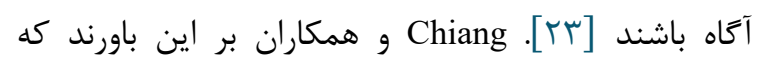

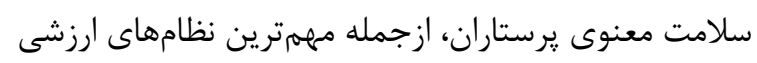

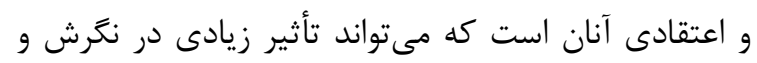

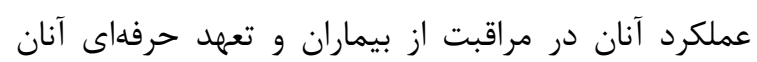
داشته باشد [rr]

با وجود نقش مهم سلامت معنوى و حساسيت اخلاقى در

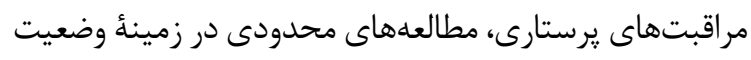

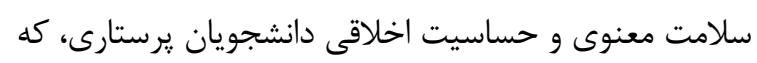

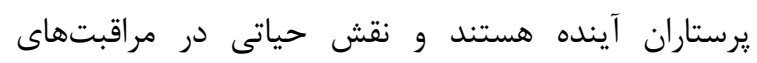

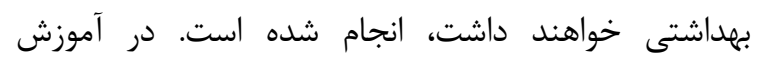

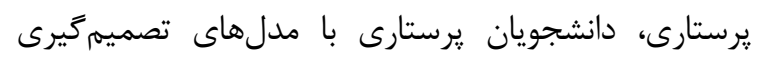

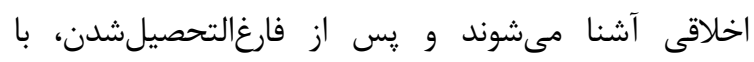

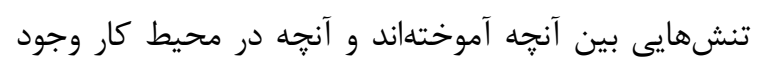

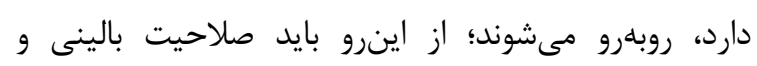

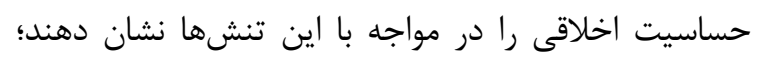

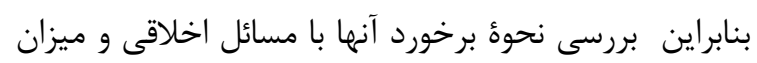

مىشود و آنها را از زاوئ اخلاق مىبيند. براى اين كار بايد

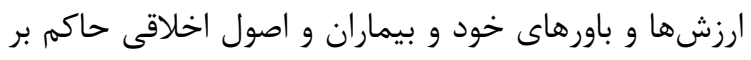

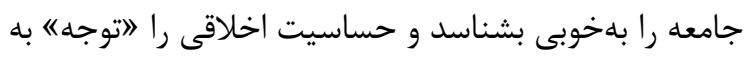

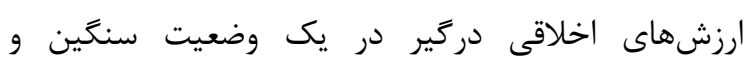
خودآكاهى از نقش و مسئوليت خويش در اين وضلى وضعيت دريت بداند.

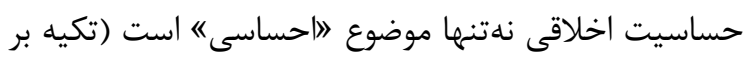
احساسات براى شناسايى يك دركيرى اخلاقى است) بلكه

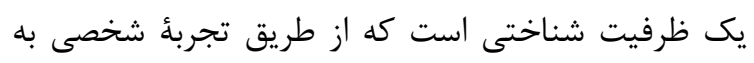

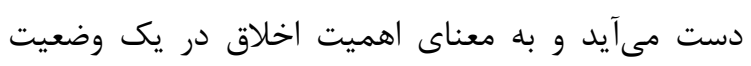

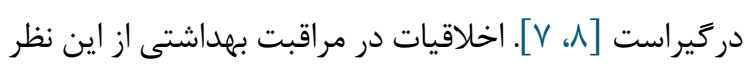

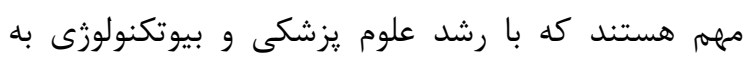

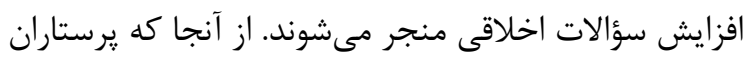

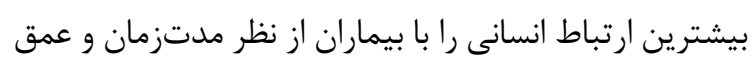

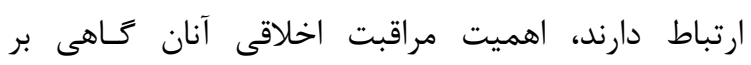

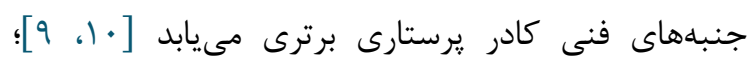

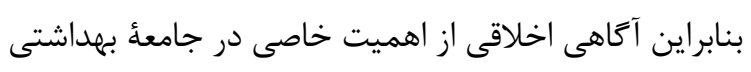

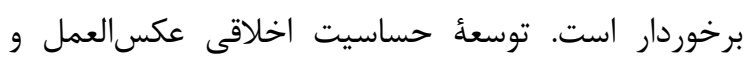

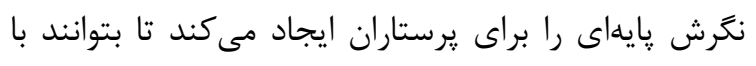

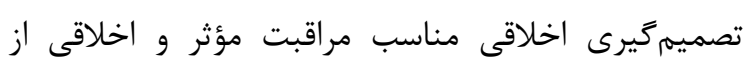

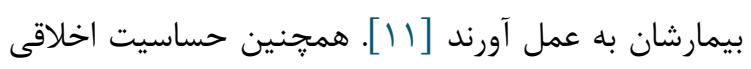

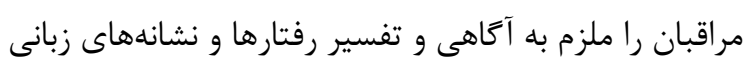

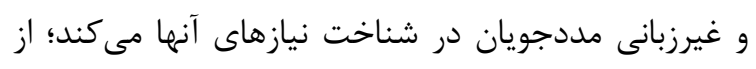

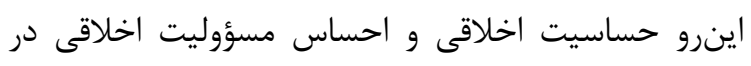

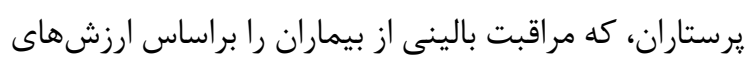

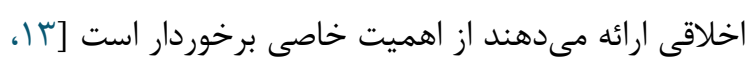

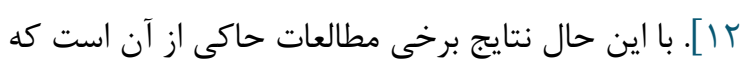

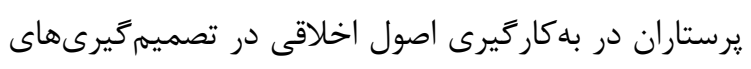
خود، ضعيف هستند و از حساسيت اخلاقى كافى برخوردار

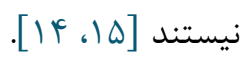
مرورى بر مطالعات نشان مىدهد كه در تعريف يرستار

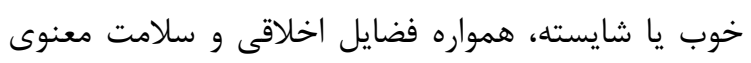

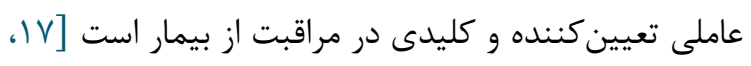

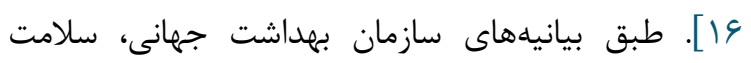

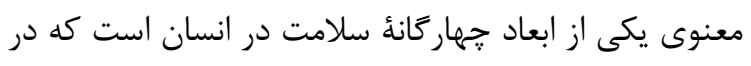

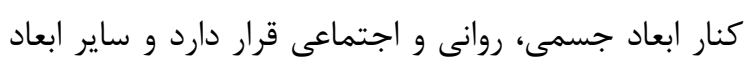

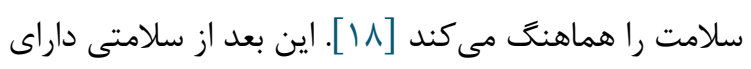

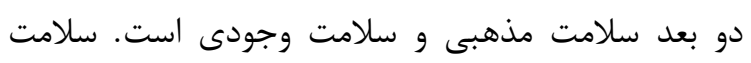

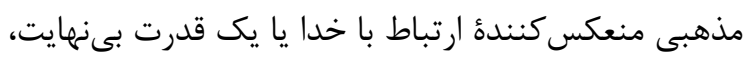


كردند [rQT]. سيس اين يرسشنامه در كشورهاى مختلف از جمله ايران استفاده شد. اعتبار اين يرسشنامه در ايران آنان

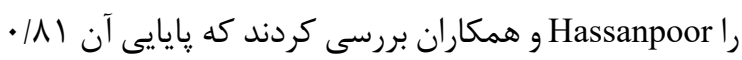

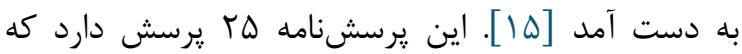
وضعيت تصميمكيرى اخلاقى يرستاران را هنتام ارائٔ

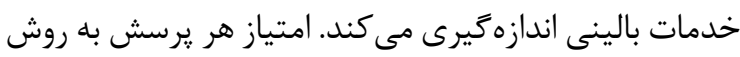

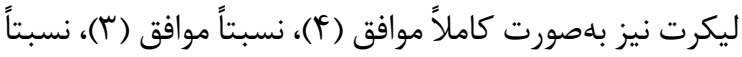

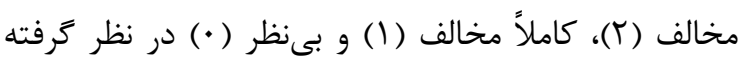
شده است. بيشترين امتياز · · إ و كمترين امتياز صفر است.

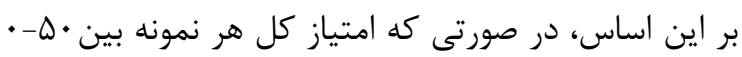

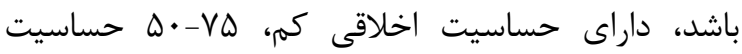

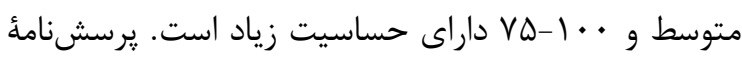

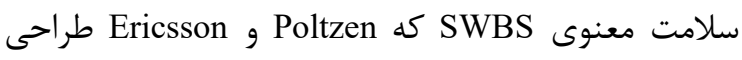

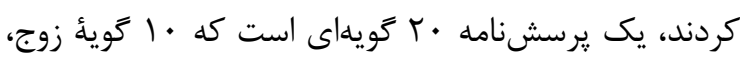

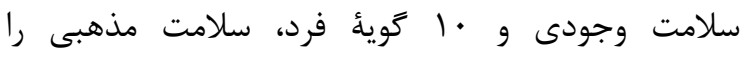

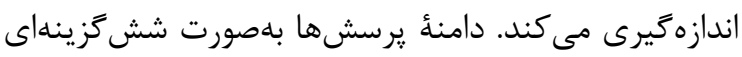

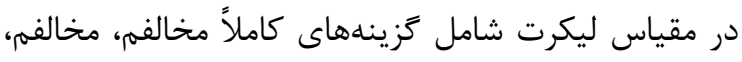

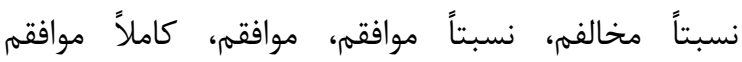

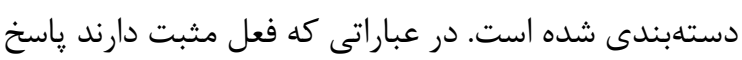

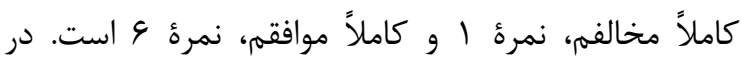
يرسشهايى كه فعل منفى دارند ياسخ كاملاً مخالفم نمرة

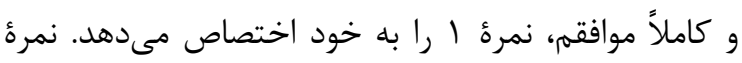

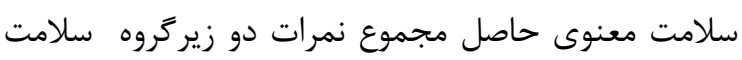

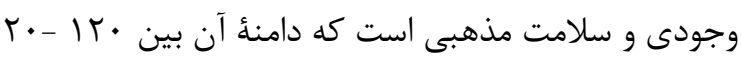

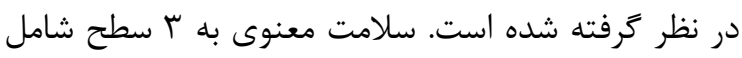

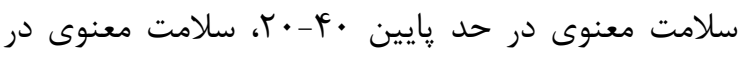

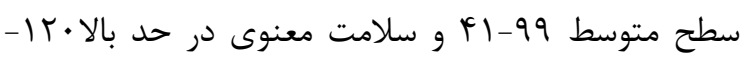

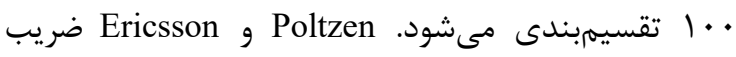

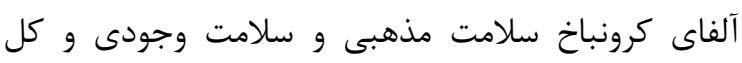

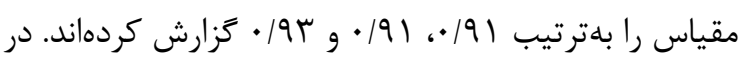

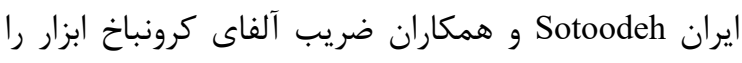

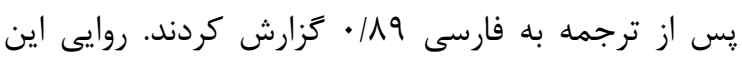

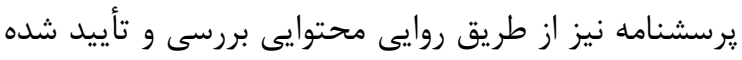

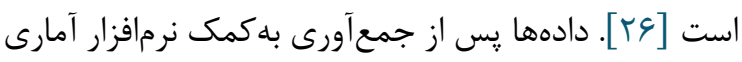

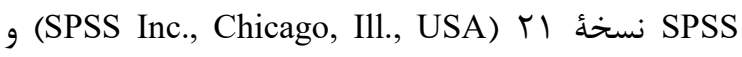

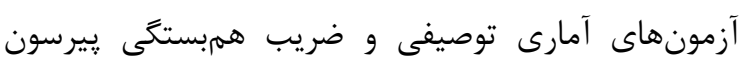
تجزيه و تحليل شدند. كفتنى است سطح معنى دارى دار در اين

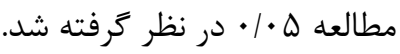

حساسيت اخلاقىشان در حيطؤ كار برستارى و تعيين عوامل

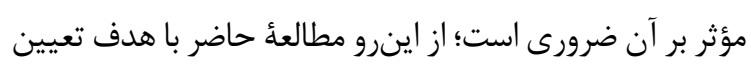

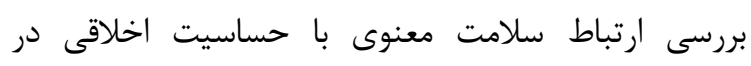
دانشجويان يرستارى انجام شده است.

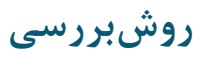

يزوهش حاضر مطالعهاى توصيفى- مقطعى از نوع

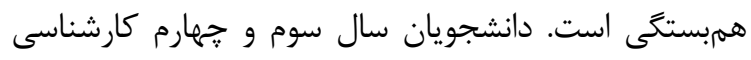

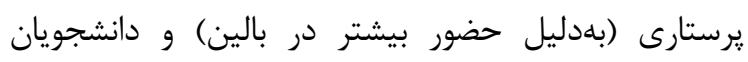
كارشناسى ارشد يرستارى دانشكدة يرستارى مامايى همدان در نيمسال اول تحصيلى

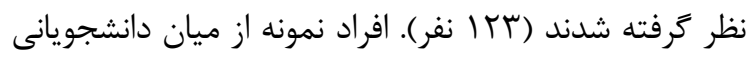

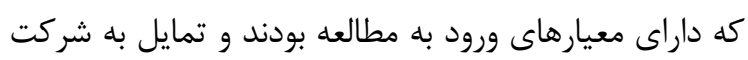

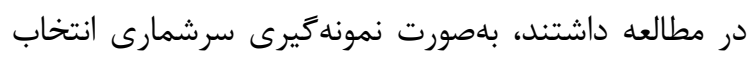

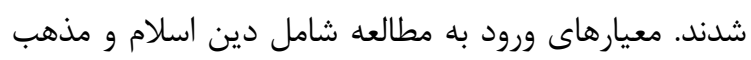

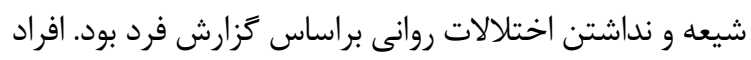

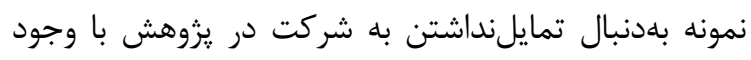

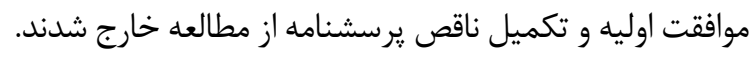

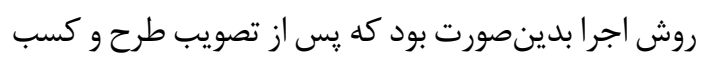

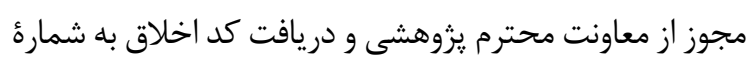

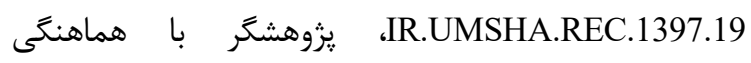
دانشكده و در زمان مناسب، يرسشنامهها را توزيع كرد قبل

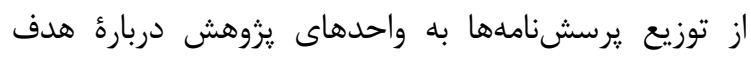

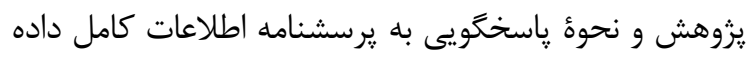

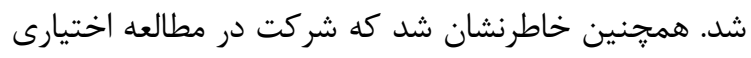

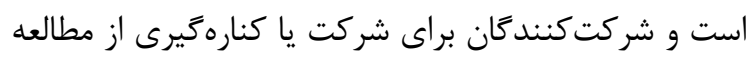

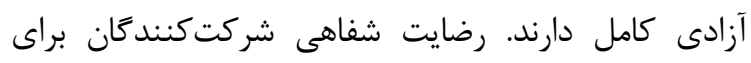

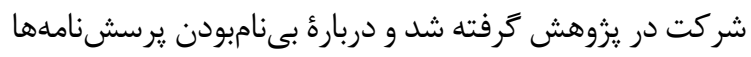

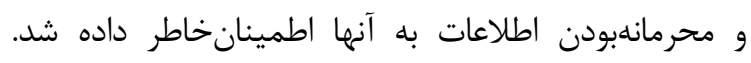

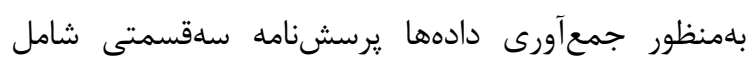

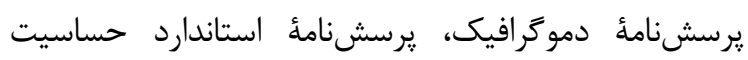

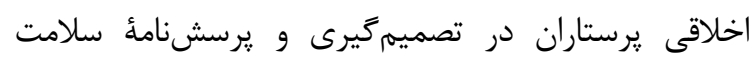
معنوى، توسط افراد نمونه تكميل شد. درى تصديم

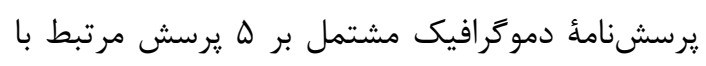

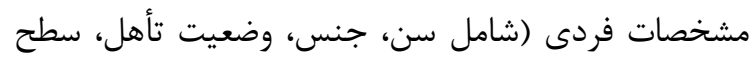

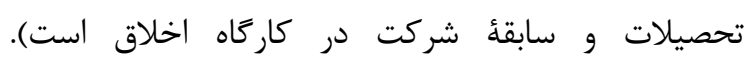

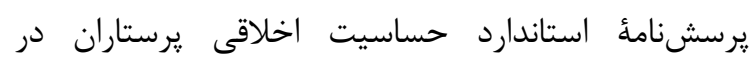

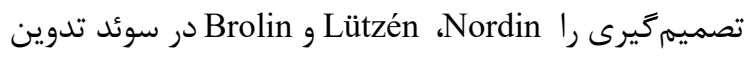




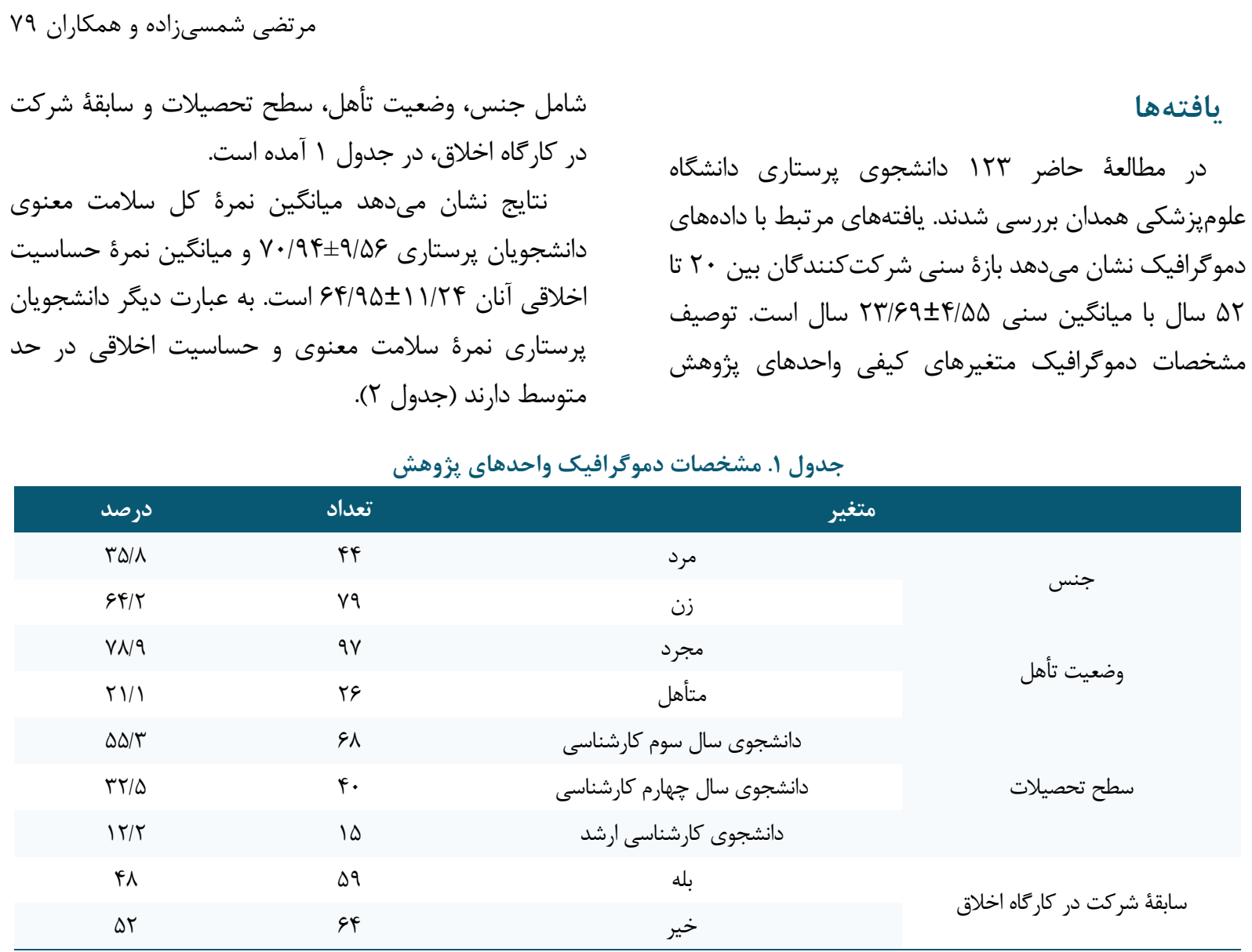

جدول r. شاخصهاى براكندگى متغير سلامت معنوى و ديسترس اخلاقى :برستاران

\begin{tabular}{|c|c|c|c|c|}
\hline دامنهُ نمرات كسبشده & دامنهُ نمرات قابل كسب & انحراف معيار & ميانَّين نمره & متغير \\
\hline$|\cdot-\Delta|$ & $1 \cdot-9$. & $\Delta / \Delta F$ & ( & سلامت مذهبى \\
\hline $1 \cdot-48$ & $1 \cdot-9$. & D/rT & my/gN & سلامت وجودى \\
\hline$r \cdot-9 r$ & $r \cdot-1 r \cdot$ & $9 / \Delta ৭$ & $V \cdot 194$ & سلامت معنوى كل \\
\hline$\cdot-94$ & $1 \cdots-$ & $11 / T^{F}$ & $94 / 90$ & حساسيت اخلاقى \\
\hline
\end{tabular}

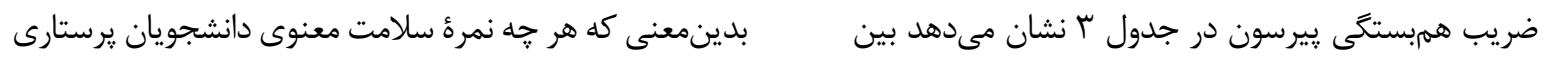

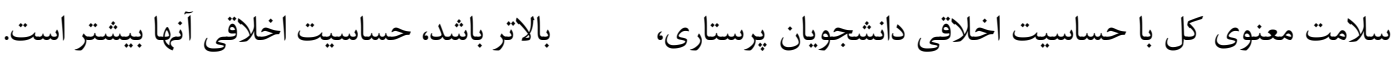

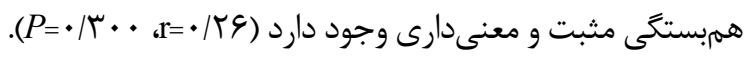

جدول س. جدول همبستكى پِيرسون بين متغيرهاى سلامت معنوى با حساسيت اخلاقى دانشجويان يرستارى

\begin{tabular}{|c|c|c|c|c|}
\hline p & $r$ & $r$ & 1 & متغير \\
\hline- & - & - & 1 & سلامت مذهبى \\
\hline - & - & 1 & 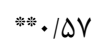 & سلامت وجودى \\
\hline- & 1 & $* * 1 \mathrm{~N}$ & $* * 1 / 9$ & سلامت معنوى كل \\
\hline 1 & $* * / r q$ & $* * * / T V$ & $* / 19$ & حساسيت اخلاقى \\
\hline \multicolumn{5}{|c|}{ 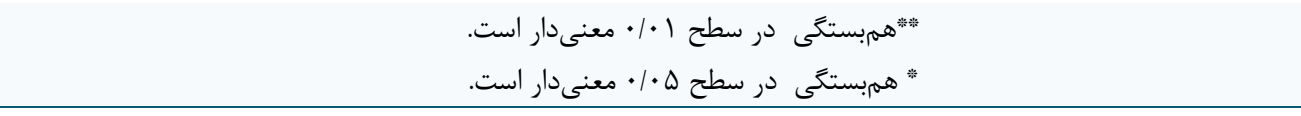 } \\
\hline
\end{tabular}


•1 بررسى ارتباط سلامت معنوى با حساسيت اخلاقى دانشجويان برستارى

يرستارى سلامت معنوى ارتباط مثبتى با اضطراب [•r]]،

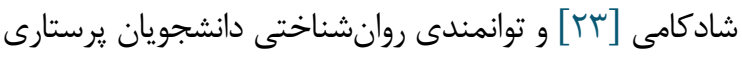

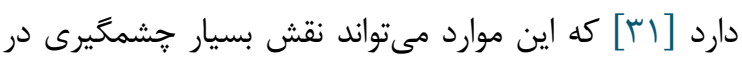
افزايش حساسيت اخلاقى دانشجويان يرستارى داشته باشد. با توجه به نقش سلامت معنوى بر حساسيت اخلاقى دانشجويان يرستارى، تقويت اين بعد از سلامت افراد از طريق باديق

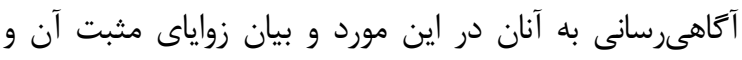

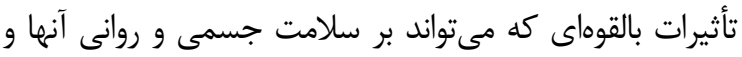

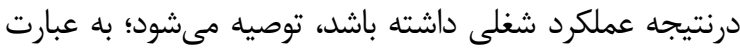

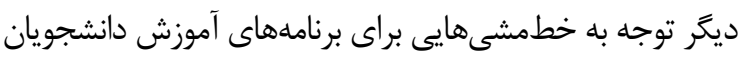

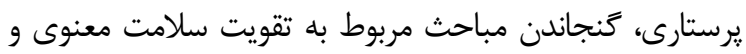

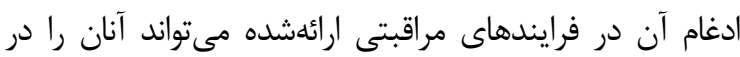
حصول آمادگى لازم در كنترل تصميمَيرى اخلاقى يارى كند.

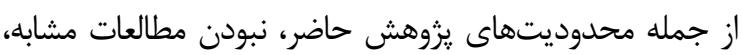

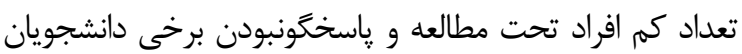

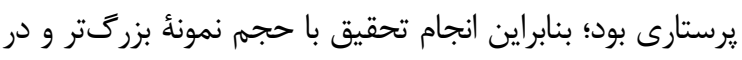

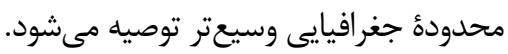

$$
\text { نتيجه }
$$

با توجه به نقش سلامت معنوى بر حساسيت اخلاقى

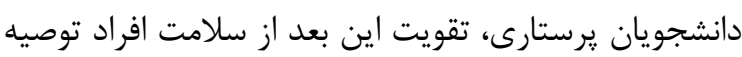

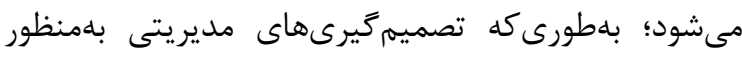

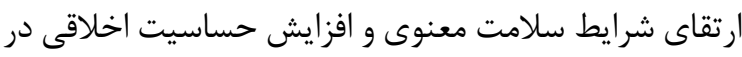
دانشخاهها براى دانشجويان يرستارى مىتواند مؤثر باشد.

$$
\text { سياسگزارى }
$$

مطالعة حاضر حاصل طرح تحقيقاتى به شمارئ TSII I F F VAF

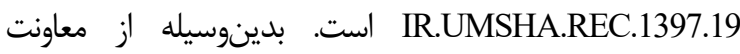
يروهشى دانشكاه علوميزشكى همدان و شركت كنندكان بامنظور

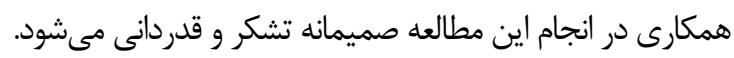

$$
\text { تعارض در منافع }
$$

بين نويسندًان هيجَّونه تعارضى در منافع وجود ندارد

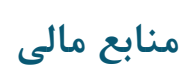

منابع مالى اين مطالعه توسط نويسندكان تامين شده است.
بحث

در مطالعأ حاضر، ارتباط سلامت معنوى با حساسيت اخلاقى دانشجويان يرستارى بررسى شد. بلطوركلى يافتهها نشان مى دهاطد

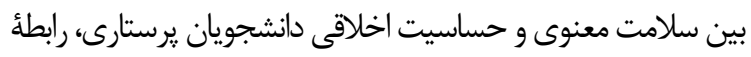

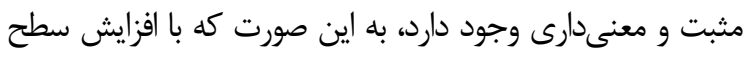

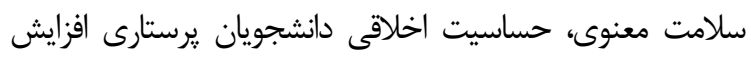

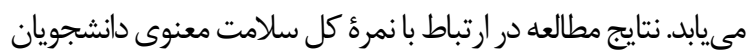

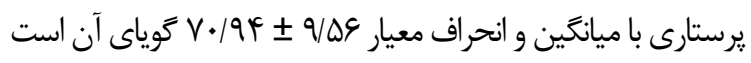
كه دانشجويان يرستارى از سلامت معنوى متوسطى برخوردار

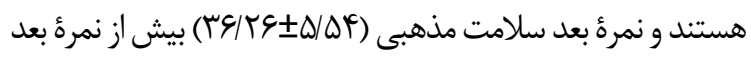

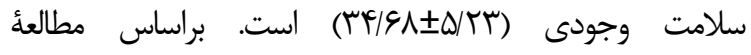
Hajimohammad g Adib-Hajbaghery ،Gharehboghlou دانشجويان يرستارى شهر قم از سلامت معنوى متوسطى Hoseini

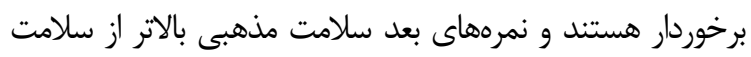

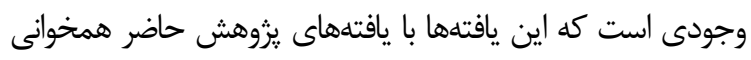

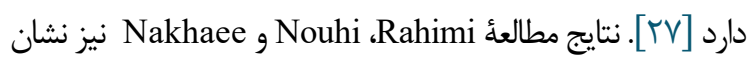

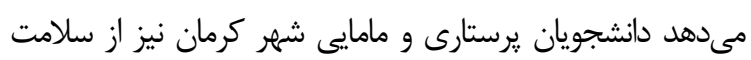

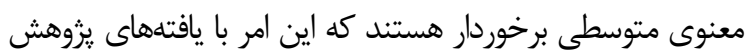

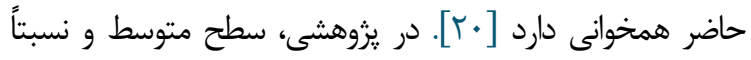

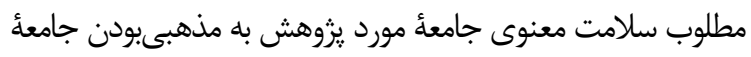

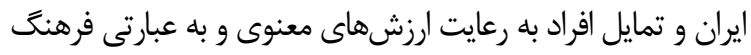

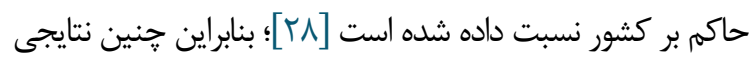

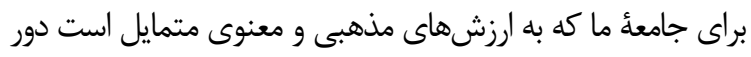

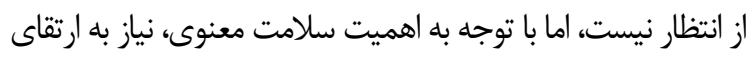

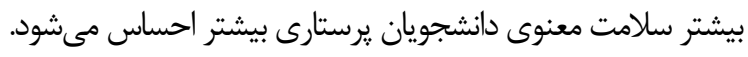

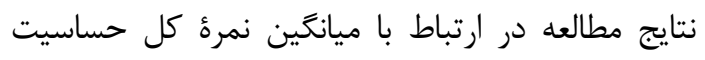

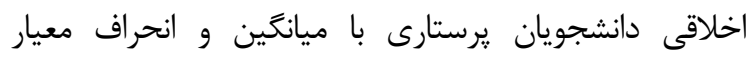

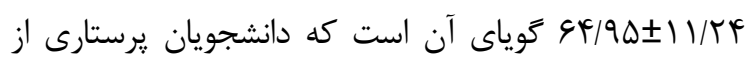
حساسيت اخلاقى متوسطى برخوردار هستند. ميزان حساسيت

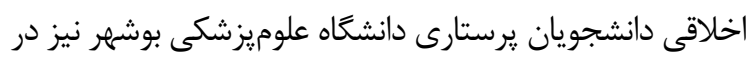

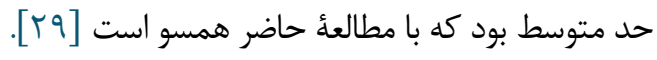

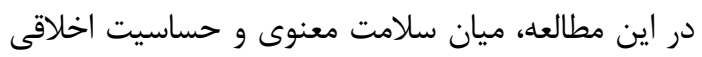

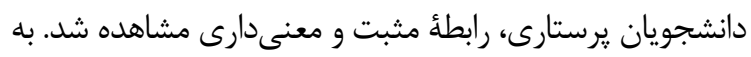
اين صورت كه با افزايش سطح سلامت معنوى، حساسيت

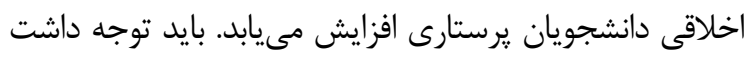

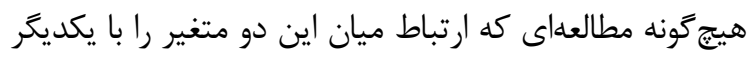

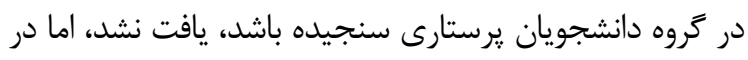
مطالعات نزديك به موضوع مىتوان اشاره كرد كه در دانشجويان 


\section{References}

1. Farasatkish R, Shokrollahi N, Zahednezhad H. Critical care nurses' moral sensitivity in Shahid Rajaee Heart Center Hospital. Iranian Journal of Cardiovascular Nursing. 2015;4(3):36-45.

2. Udomluck S, Tonmukayakul O, Tiansawad S, Srisuphan W. Development of Thai nurses' caring behavior scale. Pacific Rim International Journal of Nursing Research. 2010;14(1):32-44.

3. Mohammadi S, Borhani F, Roshanzadeh M. Moral sensitivity and delivering bad news skills: A study on critical care unit nurses. Medical Ethics Journal. 2016;10(37). [DOI:10.21859/mej-10377]

4. Milliken A. Nurse ethical sensitivity: An integrative review. Nursing ethics. 2018 May;25(3):278-303. [DOI:10.1177/0969733016646155] [PMID]

5. Ohnishi K, Ohgushi Y, Nakano M, Fujii H, Tanaka H, Kitaoka K, Nakahara J, Narita Y. Moral distress experienced by psychiatric nurses in Japan. Nursing Ethics. $2010 \quad$ Nov;17(6):726-40. [DOI:10.1177/0969733010379178] [PMID]

6. Lützén K, Dahlqvist V, Eriksson S, Norberg A. Developing the concept of moral sensitivity in health care practice. Nursing ethics. 2006 Mar;13(2):187-96. [DOI:10.1191/0969733006ne837oa] [PMID]

7. Kuilman L, Jansen GJ, Mulder LB, Middel B, Roodbol PF. Re-assessing the validity of the Moral Sensitivity Questionnaire (MSQ): Two new scales for moral deliberation and paternalism. Journal of Evaluation in Clinical Practice. 2020 Apr;26(2):659-69. [DOI:10.1111/jep.13353] [PMID]

8. Khalighi E, Solaimanizadeh L, Borji M, Tarjoman A, Soltany B, Zareie F. Investigating relationship between religious commitment and moral sensitivity in nurses working in ICU. BMC research notes. 2020 Dec 1;13(1):41. [PMID] [PMCID] [DOI:10.1186/s13104-020-4912-x]

9. Yeom HA, Ahn SH, Kim SJ. Effects of ethics education on moral sensitivity of nursing students. Nursing ethics. 2017 Sep;24(6):644-52. [DOI:10.1177/0969733015622060] [PMID]

10. Borhani F, Abbaszadeh A, Mohamadi E, Ghasemi E, Hoseinabad-Farahani MJ. Moral sensitivity and moral distress in Iranian critical care nurses. Nursing ethics. 2017 [DOI:10.1177/0969733015604700] [PMID]

11. Kim HR, Ahn SH. Moral sensitivity and moral distress among Korean hospital nurses. Korean Journal of $\begin{array}{lll}\text { Medical Ethics. 2010;13(4):321-36. } & \end{array}$ [DOI:10.35301/ksme.2010.13.4.321]

12. Decety J, Howard LH. The role of affect in the neurodevelopment of morality. Child Development Perspectives. $2013 \quad$ Mar;7(1):49-54. [DOI:10.1111/cdep.12020]

13. Decety J, Michalska KJ, Kinzler KD. The contribution of emotion and cognition to moral sensitivity: a neurodevelopmental study. Cerebral cortex. 2012 Jan 1;22(1):209-20. [DOI:10.1093/cercor/bhr111] [PMID]

14. Baghaei R, Zareh H, Aminolshareh S, Moradi Y. The ethical sensitivity of nurses in decision making in Ayatollah Taleghani Hospital, 1391. The Journal of Urmia Nursing and Midwifery Faculty. 2014;11(11):900-6.

15. Hassanpoor M, Hosseini M, Fallahi Khoshknab M, Abbaszadeh A. Evaluation of the impact of teaching nursing ethics on nurses' decision making in Kerman social welfare hospitals in 1389. Iranian Journal of Medical Ethics and History of Medicine. 2011 Oct 15;4(5):58-64.

16. Catlett S, Lovan SR. Being a good nurse and doing the right thing: A replication study. Nursing Ethics. 2011 Jan;18(1):54-63. [DOI:10.1177/0969733010386162] [PMID]

17. Begley AM. On being a good nurse: reflections on the past and preparing for the future. International Journal of Nursing Practice. 2010 Dec;16(6):525-32. [DOI:10.1111/j.1440-172X.2010.01878.x] [PMID]

18. Guilbert JJ. The World Health Report 1998-Life in the 21st Century. A Vision for All. Education for Health. 1999 Nov 1;12(3):391.

19. Atashzadeh-Shoorideh F, Abdoljabbar M, Karamkhani M, Khubestani MS, Pishgooie SA. The relationship between nurses' spiritual health and their caring behaviors. Journal of Research on Religion \& Health. 2017 Jan 30;3(1):5-15.

20. Rahimi N, Nouhi E, Nakhaee N. Spiritual health among nursing and midwifery students at kerman university of medical sciences. Journal of hayat. 2014 Mar 15;19(4):74-81.

21. Jalili F, Saeidnejad Z, Aghajani M. Effects of spirituality training on the moral sensitivity of nursing students: A clinical randomized controlled trial. Clinical Ethics. 2020 Mar;15(1):1-0. [DOI:10.1177/1477750919898346]

22. Lee E, Kim Y. The relationship of moral sensitivity and patient safety attitudes with nursing students' perceptions of disclosure of patient safety incidents: A cross-sectional study. Plos one. 2020 Jan 10;15(1):e0227585. [DOI:10.1371/journal.pone.0227585] [PMCID]

[PMID]

23. Ebadi BN, Hosseini MA, Rahgoi A, Fallahi Khoshknab M, Biglarian A. The relationship between spiritual health and happiness among nursing students. Journal of nursing education. 2017 Jan 10;5(5):23-30. [DOI:10.21859/jne-05054]

24. Chiang YC, Lee HC, Chu TL, Han CY, Hsiao YC. The impact of nurses' spiritual health on their attitudes toward spiritual care, professional commitment, and caring. Nursing outlook. 2016 May 1;64(3):215-24. [DOI:10.1016/j.outlook.2015.11.012] [PMID]

25. Lützén K, Nordin C, Brolin G. Conceptualization and instrumentation of nurses' moral sensitivity in psychiatric practice. International Journal of Methods

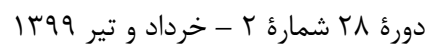$$
\text { مجله مراقبت يُرستارى و مامايى ابنسينا }
$$ 
in Psychiatric Research. 1994 Dec. [DOI:10.1037/t60329-000]

26. Sotoodeh H, Shakerinia I, Jobaneh RG, Kheyrati M, Seddigh MH. Role of Family Health and Spiritual health on Nurses Psychological Wellbeing. Medical History Journal. 2016 Oct 26;7(25):161-84.

27. Gharehboghlou Z, Adib-Hajbaghery M, Hajimohammad Hoseini M. The Relationship between Spiritual Well-Being and Depression in Nursing Students. Iran Journal of Nursing. 2016 Dec;29(103):41-50. [DOI:10.29252/ijn.29.103.41]

28. Rahimi N, Nouhi E, Nakhaee N. Spiritual well-being and attitude toward spirituality and spiritual care in nursing and midwifery students. Iran Journal of nursing. 2013 Dec;26(85):55-65.

29. Kohansal Z, Avaznejad N, Bagherian B, Jahanpour F. Evaluation of Moral Sensitivity in Nursing Students of Bushehr University of Medical Sciences in 2016. Iranian Journal of Medical Ethics and History of Medicine. 2018 Apr 15;11(1):242-52.

30. Rahimi N, Asadolahi Z, Afsharipour A. A study on the relationship between spiritual health and level of anxiety in the students of nursing and midwifery faculty. Journal of Religion and Health. 2017 Mar 10;4(2):56-64.

31. Beigi Broujeni R, Khademi Z. The relationship between spiritual well-being and psychological empowerment in nursing students. Development Strategies in Medical Education. 2015 Oct 15;2(2):7582. [마ticle] 